\title{
Igualdad, sustentabilidad y ciudadanía ecológica
}

\author{
Ramón MÁIz
}

Gerald Cohen

In memoriam

Recibido: 17 de noviembre de 2010 .

Aceptado: 15 de diciembre de 2010.

\section{RESUMEN}

Frente a la tradicional discusión contemporánea en mesas separadas de la teoría política verde y las teorías de la igualdad, se propone aquí una lectura igualitarista del principio de sustentabilidad. Junto a la responsabilidad hacia las generaciones futuras y el respeto a los límites de los ecosistemas, la sustentabilidad debe incorporar como un aspecto fundamental la igualdad de oportunidades de bienestar en el interior de cada país, así como entre Norte y Sur. La sustitución de la hipótesis de la abundancia por la hipótesis de la escasez requiere que los debates en torno a la igualdad incluyan el horizonte teórico de la sustentabilidad y sus exigencias. Todo ello se traduce en la postulación de una ciudadanía ecológico-igualitaria netamente diferenciada de la ciudadanía liberal medioambiental.

\section{PALABRAS CLAVE}

Ciudadanía ecológica, sustentabilidad, igualdad, necesidades básicas.

\begin{abstract}
In contrast with the usual separation of green political theories from theories of equality, here we propose an egalitarian understanding of the principle of sustainability. Along with responsibility towards future generations and respect for the limits of ecosystems, the concept of sustainability should include as key aspects the equality of opportunities for well-being within a country, and between north and south.The replacement of the hypothesis of abundance with one of scarcity requires a refocusing of the debates on equality to include the theoretical plane of sustainability and its requirements. This implies a concept of ecological-egalitarian citizenship that is very distinct from the idea of liberal environmental citizenship.
\end{abstract}




\section{KEY WORDS}

Ecological citizenship, sustainability, equality, basic needs.

Solo el hombre social es consciente de la esencia humana de la naturaleza, pues solo entonces se le presenta esta como aquello que lo vincula a los otros seres humanos...y de este modo se convierte la naturaleza en la base de su propia existencia humana.

Karl Marx ${ }^{1}$

Le temps du monde fini commence.

Paul Valéry ${ }^{2}$

\section{LA REFORMULACIÓN IGUALITARISTA DEL PRINCIPIO DE SUSTENTABILIDAD}

Pese a todas sus ambigüedades y usos alternativos, el principio de sustentabili$d a d^{\beta}$ retiene un importante valor político-normativo, no meramente técnico-científico. La sustentabilidad como concepto teórico se distancia notablemente del sentido burocrático y coloquial de la expresión sostenible como mera durabilidad o mantenimiento en el tiempo de determinadas cualidades, funciones o rasgos; implica la exigencia de mantener el desarrollo humano dentro de la capacidad reproductiva de los ecosistemas (carrying capacity).

Ahora bien, en la perspectiva igualitarista que proponemos en estas páginas, la sustentabilidad (a diferencia de la mera sostenibilidad), se articula conceptualmente de modo complejo mediante tres vectores fundamentales:

1 "Das mensliche Wessen der Natur ist erst da für den gesellsfatlicehn Menschen; denn erst hier ist sie für ihn da als Band mit dem Menschen, als Dasein seiner für den andren und des andren für ihn, wie als Lebenselement der menslichen Wirklichkeit, erst hier ist sie da als Grundlage seines eignen menslichen Daseins". Karl MARX, "Ökonomische-philosophische Manuskripte aus dem Jahre 1844”, en Marx-Engels Werke (MEW), vol. 40, Dietz, Berlin, 1985, p. 537. p. 923.

2 Paul VAlÉRY, Regards sur le monde actuel (1945), en CEuvres, II, Gallimard, Paris, 1960 ,

${ }^{3}$ Bryan Norton, Sustainability. A Philosophy of Adaptive Ecosystem Management, Chicago University Press, Chicago, 2005, passim. 
1) la biosfera, 2) las generaciones futuras y 3) la sociedad global. Detengámonos de modo sucinto en cada uno de ellos (Gráfico 1).

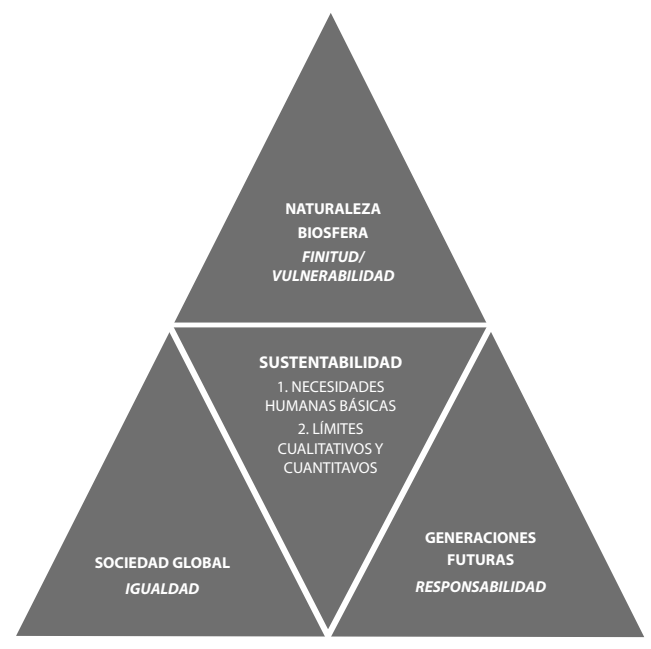

\section{Gráfico 1}

En primer lugar, sustentabilidad hace referencia a la idea de equilibrio entre la sociedad y la naturaleza, es decir, de viabilidad ecológica. Esto significa que los sistemas sociales deben reproducirse sin deteriorar irreparablemente los ecosistemas - en el sentido integral de entorno biofísico - sobre los que se apoyan.

La ecosfera o biosfera, como conjunto de ecosistemas y ámbito caracterizado por la finitud y la vulnerabilidad constituye, pues, el elemento primero de la noción de sustentabilidad. De ahí se deriva una inicial consecuencia normativa: vivir respetando los límites. Ahora bien, la sustentabilidad debe ser entendida en su sentido fuerte, esto es, más allá de la racionalidad de la economía estándar, y "formulada desde la racionalidad de esa economía de la física que es la termodinámica y de esa economía de la naturaleza que es la ecología" ${ }^{\text {". Esto se }}$ traduce en la definición del principio de sustentabilidad como aquél que demanda garantizar un nivel suficiente de capital natural. A su vez, la sustentabilidad en sentido débil solo menciona la preservación del nivel de capital total (natu-

${ }^{4}$ José Manuel NAREDO, "Sobre el origen, el uso y el contenido del término sostenible", en La Construcción de la ciudad sostenible, Habitat, Madrid, 1997, p. 8. 
ral, humano y cultivado) y presupone la progresiva sustitución del capital natural por el humano, de la naturaleza por la tecnología ${ }^{5}$.

Podríamos decir que, sin incurrir en la ensoñación arcádica de una naturaleza prístina e intocada (wilderness), la sustentabilidad en sentido fuerte se enfrenta abiertamente a la ensoñación fáustica; a la quimera de que algún día el progreso científico-técnico podrá reemplazar las funciones que realiza la naturaleza.

De forma resumida, la sustentabilidad en sentido fuerte implica varias consideraciones:

1) Se asume la existencia de naturaleza no humana (capital natural). Con ello se rechaza la tesis muy extendida de que, a estas alturas del siglo veintiuno, "la naturaleza ya no existe", "se ha disuelto", "ha desaparecido", o de que "se han abolido las distancias que nos separan del mundo natural".

2) Se postula que esta naturaleza no humana sufre presiones estructurales constantes bajo el sistema capitalista para ser reemplazada por capital no natural, de modo descontrolado, lo cual posee efectos negativos para el bienestar y la salud de los seres humanos.

3) Se defiende que al menos parte de esta naturaleza (capital natural crítico) posee un valor (intrínseco y extrínseco) que debe preservarse.

4) La sustentabilidad se formula como concepto político, no meramente económico o científico. Dadas las dimensiones que reclama, requiere a su vez un formato muy exigente y profundo de democracia. Una democracia en la que, por ejemplo, la representación se amplía para dar cabida a voces que hablan en nombre de la naturaleza no humana — pues la naturaleza habla, emite señales y por lo tanto puede y debe ser de algún modo escuchada e incluso merecedora de un cierto respeto ético ${ }^{7}$ - Junto a ello, la deliberación/inclusión se convierte en el eje de la decisión pública sobre los estilos de vida, riesgos y costes que estamos dispuestos a asumir para preservar el capital natural tanto de forma colectiva (con las editing choice policies: políticas públicas de gestión de la demanda y el consumo, por ejemplo), como individual (con nuevas pautas vitales de reducciónreciclado-reutilización).

${ }^{5}$ Eric NeumaYer, Weak versus Strong Sustainability: Exploring the Limits of Two Opposing Paradigms, Edward Elgar, London, 2003, p. 21.

${ }^{6}$ Anthony Giddens, The Politics of Climate Change, Polity, Cambridge, 2009, passim.

${ }^{7}$ Bruno Latour, Politics of Nature, Harvard University Press, Cambridge, 2004, passim. 
En segundo lugar, la sustentabilidad introduce otro elemento esencial: las generaciones futuras. Desde al menos el Informe Brundtland ${ }^{8}$, la sustentabilidad implica atender las necesidades del presente sin comprometer la capacidad de las futuras generaciones de atender las suyas. Esto se traduce en un principio de responsabilidad o de justicia intergeneracional; esto es, en la obligación moral de "no poner en peligro las condiciones para la supervivencia indefinida de la humanidad sobre la Tierra"". O lo que es lo mismo, no hipotecar ni la libertad de poder elegir entre las opciones disponibles de vida, ni la igualdad de recursos y capacidades para atender las necesidades básicas de las futuras generaciones.

Edith Weiss ha concretado, de modo inmejorable, la equidad intergeneracional. Plantea tres principios que restringen las acciones de las presentes generaciones para usar los recursos naturales y culturales de nuestro planeta: i) conservación de recursos naturales y culturales por parte de la generación presente para evitar la restricción de opciones disponibles por las futuras generaciones (conservación de opciones); ii) mantenimiento de la calidad del planeta para su disfrute futuro en condiciones no inferiores a las de la presente generación (conservación de la calidad); y iii) derecho de acceso equitativo al legado de las generaciones pasadas y preservación de este acceso a las futuras (conservación de acceso) ${ }^{10}$.

El principio de responsabilidad vincula estrechamente: 1) la biosfera como fuente de obligación ético-política por derecho propio, 2) las condiciones globales de la vida humana sobre la tierra más allá de las fronteras de los Estados nacionales y 3) la asimetría/no reciprocidad como principio de una ética y una política orientadas al futuro y en defensa de la capacidad de atender las necesidades básicas de las siguientes generaciones. Esta intuición la encontramos sintetizada de modo extraordinariamente lúcido en el libro III de El Capital de Karl Marx (1818-1883):

Ni una entera sociedad, ni una nación, ni siquiera todas las sociedades contemporáneas en su conjunto, son las propietarias de la tierra. No son más que sus meras poseedoras, sus usufructuarias, y deben, como boni patres familias, legarla a las futuras generaciones en las mejores condiciones posibles ${ }^{11}$.

\footnotetext{
${ }^{8}$ Se trata de un informe socio-económico de la ONU elaborado en 1987 por una comisión encabezada por Gro Harlem Brundtland. En este informe aparece por primera vez el término desarrollo sostenible.

${ }^{9}$ Hans Jonas, El Principio de Responsabilidad, Herder, Barcelona, 1995, p. 25

${ }^{10}$ Edith WeIss, In Fairness of Future Generations, United Nations, New York, 1989, p. 57.

11 "Selbst eine Gesellschaft, eine Nation, ja alle gleichzeitigen Gesellschaften zusammengenommen, sind nicht Eigentümer der Erde. Sie sind nur ihre Besitzer, ihre Nutzniesser, und haben
} 
En tercer lugar, a los dos elementos anteriores debe añadirse otro no menos decisivo, si bien a menudo descuidado en la teoría política verde. Se trata de la justicia social, y en concreto del principio de igualdad, no ya entre generaciones, sino en el seno de la presente generación a escala local y global. La igualdad es, en sentido estricto, constitutiva de la sustentabilidad y debe ser formulada como corrección de las desigualdades de clase que se dan dentro de cada país, pero también y en no menor medida, de las desigualdades que existen entre los países del mundo.

A diferencia de un uso muy extendido del concepto de sustentabilidad que lo reduce a la sola responsabilidad intergeneracional, este principio pone en primer plano e integra como uno de sus componentes básicos la igualdad intrageneracional en lo que concierne al reparto de recursos y capacidades; es decir, la redistribución equitativa de los beneficios que nos aporta la naturaleza así como de los niveles de daño admisibles tanto a nivel global como local (contaminación, agotamiento de recursos renovables y no renovables, pérdida de biodiversidad, problemas de salud, etc.).

Debe notarse la estrecha vinculación de este factor con el anterior: la obligación de proteger las opciones de vida de las generaciones futuras requiere restricciones, redefinición de límites y de necesidades, gestión de la demanda, etc. Acciones que reclaman procedimental y sustantivamente la redistribución de recursos y de costes en la presente generación.

El argumento es que desarrollo insostenible en el futuro y desigualdad social en el presente constituyen dos síntomas del mismo síndrome: el sobrecrecimiento. Dicho de modo sumario, el modelo de desarrollo capitalista es a la vez ecológicamente insostenible y socialmente injusto. Cualquier política de lucha contra el crecimiento insostenible, en cuanto se traduce de modo inevitable en la imposición de límites — que, en su estándar actual, no es posible universalizarya sea en el consumo de recursos renovables y no renovables o en la generación de residuos, resulta injustificable sin considerar la redistribución de la riqueza existente.

A la vista del olvido generalizado de este factor, tanto en las políticas ambientalistas como en buena parte del movimiento ecologista, sorprende recordar que en el mencionado Informe Brundtland ya se aludía a esta dimensión igualitaria:

sie als boni patres familias den nachfolgenden Generationen verbessert zu hinterlassen". Karl MARX, Das Kapital (1894), Dritter Band MEW Band 25, Dietz, Berlin, 1964, p. 784. 
Incluso la más estrecha interpretación de sostenibilidad física implica una preocupación por la equidad entre generaciones, una preocupación que debe lógicamente extenderse a la equidad dentro de cada generación ${ }^{12}$.

Y es que las interacciones sociopolíticas y ecológicas son, a estos efectos, inmediatas y se implican mutuamente. Relacionarse con el mundo natural de modo viable y dentro de ciertos límites no es suficiente para asegurar la sustentabilidad individual y colectiva de los seres humanos. Es preciso incluir en un lugar prioritario la lucha contra la marginación social. Este objetivo implica prestar atención a la especial vulnerabilidad de amplios sectores de la población, dentro de cada país y en la sociedad global, que están sumidos en la pobreza y se ven afectados por la desigual distribución de los beneficios de la naturaleza y del desarrollo. Asimismo conlleva atender al desigual reparto de los daños ocasionados tanto por la naturaleza (terremotos, tsunamis, etc.) como por el sobredesarrollo (contaminación, calentamiento global y sus secuelas). Todo ello implica asumir de una vez por todas que los procesos de toma de decisiones afectan de modo capital a la sustentabilidad de los resultados; es decir, a la calidad del bienestar humano y de los ecosistemas en los que descansa ${ }^{13}$.

Existe otra razón fundamental para que, en contra de lo que se ha sostenido desde perspectivas liberales de capitalismo verde, debamos argumentar en favor de un nexo teórico entre sustentabilidad y justicia social igualitaria. El principio de sustentabilidad, como decíamos, debe ser concretado de forma democrática en cambios estructurales y en políticas públicas en los ámbitos local, nacional, europeo e internacional, lo cual requiere que en esos espacios políticos se den las condiciones necesarias para que haya una comunicación no distorsionada y una deliberación libre de coacciones y de asimetrías en cuanto a la disposición de recursos esenciales que pudieran sesgar o hipotecar sus resultados.

La relación de una comunidad con su medio ambiente, los recursos empleados, el modelo energético y de transporte, los riesgos que deben ser asumidos o evitados, la elección de las políticas de gestión de la demanda o la responsabilidad hacia las generaciones futuras, todo ello son decisiones que deben ser adoptadas democráticamente por cada comunidad. Esto último es particularmente

12 "Even the narrow notion of physical sustainability implies a concern for social equity between generations, a concern that must logically be extended to equity within each generation". Informe Bruntland (1987), p. 43. Citado en John DrYZeK, The Politics of The Earth, Oxford University Press, Oxford, 1997, p. 132.

${ }^{13}$ W. Neil Adger, Andrew Jordan, Governing Sustainability, Cambridge University Press, Cambridge, 2009, p. 6. 
relevante, toda vez que en estas decisiones de política medioambiental no solo están presentes aspectos referidos a los requisitos de procedimiento democrático, tales como información, capacidad de influencia, participación, control y responsabilidad; también están en juego valores de identidad colectiva (communityidentity values) como la cultura, el paisaje, los recursos o los modos de vida, de desarrollo y de modelo territorial; pues toda comunidad posee una dimensión a la vez social y natural que despliega su vida en un medio ambiente específico con el que interacciona ${ }^{14}$.

\section{NECESIDADES BÁSICAS Y LÍMITES DE LOS ECOSISTEMAS}

La inclusión de la igualdad como elemento central de la sustentabilidad, conjuntamente con la finitud/vulnerabilidad de la biosfera y la responsabilidad hacia las futuras generaciones, resulta decisiva para dar cuenta de dos conceptos fundamentales que deben ser objeto de riguroso debate normativo: 1) necesidades básicas y 2) límites del crecimiento. Veámoslos brevemente.

El concepto de necesidades básicas articula de forma inseparable la igualdad en las futuras y en las presentes generaciones. Si bien resulta imposible adelantar los valores y preferencias de los seres humanos venideros, sí podemos discutir con fundamento qué bienes básicos les resultarán necesarios para que desarrollen oportunidades iguales a las nuestras. La sostenibilidad, en este orden de cosas, puede ser interpretada como igual libertad de oportunidades para las generaciones futuras. Ahora bien, esto significa modificar un criterio tosco de mera satisfacción de preferencias dadas (necesidades sociales, esto es, socialmente condicionadas por el actual modo de vida) y adoptar una perspectiva de necesidades no instrumentales, entendiendo por tales aquellas que atienden al mantenimiento de condiciones que son necesarias para una vida humana digna de tal nombre.

Len Doyal e Ian Gough han señalado que, para ello, puede asumirse la existencia de un mínimo de necesidades humanas objetivas entendidas como fines y estrategias para alcanzar una vida digna, como precondiciones para la prosecución de fines y la participación social. La desigual carencia de satisfacción de esas necesidades básicas provocaría daños y resulta normativamente injustificable. Así, por ejemplo, supervivencia/salud física (ausencia de enfermedad seria) y autonomía personal (la posibilidad de realizar elecciones informadas sobre qué

\footnotetext{
${ }^{14}$ NORTON, Sustainability, p. 371.
} 
hacer y cómo hacerlo), constituyen las necesidades básicas fundamentales para toda acción humana y en toda cultura ${ }^{15}$.

Sin embargo, también en este concepto debe evitarse todo rastro de esencialismo. Las necesidades humanas básicas, a diferencia de los más objetivos requerimientos del organismo (para una vida saludable, por ejemplo), nunca son necesidades en estado puro. Están abiertas a interpretaciones culturales y opciones políticas diversas. Esta consideración impide que, como alternativa al consumismo e insaciabilidad del mercado, se puedan contraponer ciertas necesidades universales y auténticas, fuera de toda discusión y válidas para todos los seres humanos ${ }^{16}$.

Distinguir entre "necesidades verdaderas" y "falsas" (Herbert Marcuse (1898-1979)) presenta serios problemas por aplicar criterios objetivos a una materia afectada por el pluralismo de valores. Sin embargo, el debate sobre las que Marx llamaba "necesidades fin en sí" o "necesidades necesarias", o Agnes Heller "necesidades radicales" 17 , constituye un ámbito central de la más amplia discusión sobre la sustentabilidad. Por ejemplo, la cuestión del tiempo de trabajo/tiempo de ocio, en conexión con la distinción entre desarrollo/crecimiento ${ }^{18}$, constituye un venero de no poco interés tanto en las políticas ecológicas de la gestión de la demanda, como en la invención de nuevos hábitos y estilos de vida sustentables. Más aún en un mundo dominado por los mecanismos de mercado que, reduciendo nuestra condición de ciudadanos a la de consumidores, nos impulsan a desear lo que no necesitamos y a no desear lo que necesitamos.

Esto apunta a una interesante conexión teórica con la noción de capacidades humanas y funciones humanas básicas de Martha Nussbaum y de Amartya Sen. La distinción que realiza Sen, por ejemplo, entre opulencia (disponibilidad de recursos), capacidades (lo que se puede lograr mediante elección), funcionamientos (la libertad de elegir una forma de vida) y ventajas (la oportunidad real de alcanzar determinado bienestar), permite conectar el debate sobre la iigualdad de qué? desde la prioridad otorgada a las capacidades; con la presente y con las futuras generaciones. Así, la insistencia en capacidades más que en bienes (considerados como meros portadores de aquellas) y la ausencia de referencias sustantivas al medio ambiente, lo cual debilita la conexión igualdad/sustentabili-

${ }^{15}$ Len Doyal, Ian Gough, A Theory of Human Need, MacMillan, London, 1991, passim.

${ }^{16}$ Kate SOPER, What is nature?, Blacwell, Oxford, 1995, passim.

${ }^{17}$ Agnes Heller, Teoria de las necesidades en Marx, Península, Barcelona, 1978, passim.

${ }^{18}$ Marx ya aludió a esta cuestión en el Libro III de El Capital, recordando el lema: "Wealth is disposable time and nothing more". [La riqueza es tiempo desechable y nada más]. 
dad $^{19}$, permite, sin embargo, enlazar (la lucha por) la igualdad en la presente generación con (la defensa de) la igualdad en las generaciones futuras en cuanto a oportunidades reales, en el sentido que luego veremos ${ }^{20}$.

Además, una noción como la propuesta por Sen - los seres humanos como portadores no solamente de necesidades pasivas, sino de valores activos - abre la puerta a situar en primer plano la dimensión política de la razón pública, las emociones, la elección y la participación. Desde un antropocentrismo débil, que evita a la vez tanto el naturalismo esencialista del biocentrismo, nivelador de los animales y los humanos, como el paradigma de explotación sin límites de la naturaleza, inseparable de un antropocentrismo fuerte, se considera que más que de un estado de naturaleza prístino e intocado a preservar, el valor del medio ambiente deriva de lo que significa para los seres humanos. Por eso, más allá de toda autoevidente aserción óntica, incluso el que consideramos irrenunciable valor intrínseco de la naturaleza no existe de forma objetiva, independiente de la contingencia y la indeterminación de la acción política con la que la naturaleza es interpretada, valorada, concretada y contestada ${ }^{21}$.

De este modo, el valor del medio ambiente, por una parte, consiste en la urdimbre vital y las decisivas oportunidades que proporciona a los seres humanos; pero por otra constituye, a su vez, un resultado performativo de la acción. Es decir, es el producto no solo de una protección pasiva, sino también de una intervención activa, habida cuenta que "el desarrollo es un proceso de apoderamiento, y ese poder puede ser usado para preservar y enriquecer el medioambiente, y no solo para destruirlo"22.

Sen postula, criticando a Robert Solow, que el fulcro de las vidas humanas no reside solamente en el estándar de vida y la satisfacción de necesidades, sino en la libertad de que disfrutan los individuos (incluida la libertad de satisfacer las necesidades). A partir de todo lo antedicho, podríamos reformular la sustentabilidad como la preservación o ampliación de las libertades reales sustantivas y de la igual capacidad de los seres humanos presentes sin comprometer la capacidad

\footnotetext{
${ }^{19}$ Andrew Dobson, Justice and the Environment, Oxford University Press, Oxford, 1998, p. 133.

${ }^{20}$ Andrew Mason, Levelling the Playing Field. The Ideal of Equal Opportunity, Oxford University Press, Oxford, 2006, passim.

${ }^{21}$ NorTOn, Sustainability, p. 373.

22 "Development is fundamentally an empowering process, and this power can be used to preserve and enrich the environment, and not only to decimate it". [El desarrollo es fundamentalmente un proceso de empoderamiento, y este poder se puede utilizar para preservar y enriquecer el medio ambiente, y no únicamente para diezmarlo]. Amartya SEN, The Idea of Justice, Penguin, New York, 2009, p. 249.
} 
de las generaciones futuras. En este sentido, asumir la perspectiva de las capacidades y desplazar la atención de los medios a las oportunidades reales proporciona una nueva métrica tanto para la igualdad en el seno de la presente generación, como entre generaciones presentes y futuras. Con ello no solo es posible repensar la pobreza como discapacidad (y la discapacidad como pobreza) y atender de modo prioritario las necesidades básicas de alimento, vivienda, sanidad y educación. Además permite realizar una relectura del principio de sustentabilidad más abiertamente política.

La razón es clara, no se pueden fijar los criterios de sustentabilidad sin tomar en consideración el pluralismo de valores en conflicto y la necesaria deliberación pública democrática para su esclarecimiento ${ }^{23}$. Necesidades básicas y capacidades se muestran, así, como conceptos alternativos a los de necesidades instrumentales o sociales y de recursos/bienes para fundamentar los criterios intrageneracionales e intergeneracionales de redistribución.

En lo que respecta a la idea de límites, su centralidad no deja lugar a dudas, toda vez que sustentabilidad implique precisamente vivir dentro de los límites de los ecosistemas: la autolimitación y suficiencia ${ }^{24}$. La idea esencial aquí es que lo que extraemos de la biosfera (energía, recursos, materias primas) y lo que desprendemos en ella (residuos, calor) debe estar dentro de los límites de reproducción del ecosistema. La imposibilidad de crecimiento material infinito en una biosfera finita se traduce en la crítica radical de la hipótesis de la abundancia desde un horizonte de escasez. $\mathrm{Y}$ en definitiva nos conduce a repensar el desarrollo al margen del mero crecimiento, desde las "constricciones estructurales que para las acciones y los proyectos humanos se derivan de la finitud y vulnerabilidad de la biosfera, del carácter entrópico del universo y de las características del ser humano"25.

La alternativa hipótesis de la escasez — "no hay de todo para todos"reclama de suyo la estrecha vinculación del principio de la igualdad al de la sustentabilidad por dos motivos:

1) En primer lugar, porque un modelo de desarrollo capitalista de las fuerzas productivas que lo son también destructivas, como ya supo ver incluso el productivista Marx en La Ideología Alemana ${ }^{26}$, y donde la desigualdad funciona como incentivo estructural de todo el sistema, aboca al sobredesarrollo y al con-

${ }^{23}$ Ibid., p. 263.

${ }^{24}$ André Gorz, Ecologica, Galilée, Paris, 2008, passim.

${ }^{25}$ Jorge Riechmann, Un mundo vulnerable, Catarata, Madrid, 2000, p. 55.

${ }^{26}$ Manuel SACRISTÁN, Pacifismo, ecologismo y política alternativa, Península, Barcelona, 1987, p. 135. 
siguiente estrechamiento de oportunidades de las generaciones futuras.

2) En segundo lugar, porque ante un horizonte de escasez, perdida toda fe en la abundancia futura, las desigualdades resultan aún más intolerables si cabe, a la vez que se vuelven más perentorios los problemas de la redistribución: ¿entre quienes? y ¿de qué? 27 .

\section{El RELIEVE NORMATIVO DE LA HUELLA ECOLÓGICA}

La reconstrucción igualitarista de la sustentabilidad apuntada en las páginas anteriores tiene su traducción política en la redefinición del concepto de ciudadanía como ciudadanía ecológica. La propia naturaleza política de la sustentabilidad demanda su determinación democrática; pero, al mismo tiempo, las desigualdades estructurales que existen entre los ciudadanos suponen un escollo para ello.

Por esta razón la huella ecológica deviene un concepto muy útil como operacionalización distributiva de la sustentabilidad, entendida esta como un modo de vida viable, esto es, equitativo - intra e intergeneracionalmente - y dentro de los límites de la naturaleza. La huella ecológica, en su versión básica, consiste en una medida de la carga impuesta por una determinada población sobre la naturaleza. En concreto, representa la porción de tierra productiva necesaria para mantener los actuales niveles de consumo de recursos y de generación de residuos por parte de una población específica (individuos o comunidades) ${ }^{28}$. La huella ecológica permite comprobar el patente exceso que las actuales pautas de desarrollo implementan sobre la biocapacidad del planeta. También permite valorar las dimensiones de la deuda o brecha ecológica, entendida esta como el diferencial entre el impacto de la tecnosfera sobre la biosfera. Al mismo tiempo, este concepto se formula desde la perspectiva de que la situación es reversible y puede cambiarse mediante políticas que reduzcan el exceso de ocupación de espacio medioambiental (deuda ecológica) en los diferentes aspectos que lo causan (tamaño de la población, consumo individual, generación de residuos, etc.).

${ }^{27}$ Véase las obras de Félix Ovejero, Proceso abierto. El socialismo después del socialismo, Tusquets, Barcelona, 2005, passim; Gerald Allan COHEN, If you're an Egalitarian, How Come You're So Rich?, Harvard University Press, Cambridge, 2000, passim; Gerald Allan CoHEN, Rescuing Justice and Equality, Harvard University Press, Cambridge, 2008, passim.

${ }^{28}$ Mathis Wackernagel, William Rees, Our Ecological Footprint, New Society, Gabriola Island, 1996, p. 9. Nicky Chambers, Craig Simmons, Mathis WaCKernagel, Sharing Nature's Interest: Ecological Footprints as an indicator of sustainability, Earthscan, London, 2000, pp. 212217. 
La hipótesis de atribución a cada persona del planeta de una huella ecológica media ${ }^{29}$ permite: 1) por una parte, detectar la insostenible desigualdad en términos de exceso o déficit de espacio medioambiental del que disponen los diferentes seres humanos (así, un consumidor medio occidental ocupa más de seis hectáreas); y 2) por otra, permite reconstruir el concepto de ciudadanía ecológica desde una perspectiva igualitaria.

Con el apoyo del concepto de huella ecológica, la reformulación igualitarista de la idea de sustentabilidad que aquí proponemos incorpora el derecho y deber de restituir un espacio ambiental igual para todos y cada uno de los seres humanos. El instrumento de la huella ecológica permite operacionalizar y medir cuantitativa y cualitativamente los costes ambientales en términos objetivos, bien del reparto inequitativo de los beneficios de la naturaleza, por ejemplo, del exceso de consumo de recursos naturales ${ }^{30}$; o bien de la desigual distribución en la producción de residuos, por ejemplo, del desigual monto de emisiones de $\mathrm{CO}_{2}$ por persona, país y año.

La huella ecológica resulta clave para reformular el concepto de ciudadanía por varias razones:

1. Proporciona una nueva noción del espacio político como contexto de deberes y derechos cívicos, diferente al territorializado del Estado-nación tradicional, pero también del cosmopolitismo clásico ("ciudadanos del mundo")

2. Genera no solo derechos, sino muy especialmente obligaciones igualitarias: la perentoria reducción del espacio ocupado en exceso por mor de pautas de consumo/contaminación insostenibles.

3. Aporta una noción de justicia medioambiental (esto es: exigible, no graciable) que considera injusta, por desigualitaria, la sobreapropiación de espacio ambiental, tanto en lo que se refiere a la generación presente como a las generaciones futuras (reducción de oportunidades) ${ }^{32}$.

4. Genera demandas normativas de cambio estructural político y económico, reformas de alcance y profundidad de sistemas basados en una hiperocupación del espacio ambiental disponible ${ }^{33}$.

${ }^{29}$ Tras dividir la superficie de la tierra habitada por su población este valor se sitúa en torno a unas 1,5-1,7 hectáreas por persona.

${ }^{30}$ Oscar CARPINTERO, El metabolismo de la economía española. Recursos naturales y huella ecológica, Fundación Cesar Manrique, Lanzarote, 2005, passim.

${ }^{31}$ Dobson, Justice and the Environment, p. 99.

${ }^{32}$ Andrew Dobson, "Citizens, citizenship and governance for sustainability", en AdGER, JoRDAn, Governing Sustainability, p. 135.

${ }^{33}$ Martin O' Connor, Is Capitalism Sustainable?, Guilford Press, New York, 1994, passim. 
5. Requiere para su desarrollo no solo argumentos y razones sino el cultivo de un nuevo ethos, una inédita sensibilidad ambiental compartida, nuevos hábitos de vida y emociones específicas como el cuidado, la compasión o la empatía.

6. Toda vez que la huella ecológica posee una vertiente política y normativa, no exclusivamente técnica y económica, precisa para su concreción en derechos/obligaciones de una democratización de la democracia en sus cuatro dimensiones fundamentales: representación, participación, deliberación e inclusión.

\section{Principios de una CiUdadanía ecológica igualitaria}

Por todo ello la ciudadanía ecológica, reformulada desde el nuevo espacio de la huella ecológica, constituye la expresión definitiva de la insoslayable naturaleza política y democrática de la sustentabilidad, tanto en lo que se refiere a las instituciones como a los actores y su interrelación.

Ahora bien, la ciudadanía ecológica se diferencia claramente de la mera ciudadania liberal medioambiental y su "greening politics", que apenas incorpora una sustentabilidad débil como dimensión adjetiva y colateral. El dualismo conceptual que aquí proponemos (véase Cuadro 1), elaborado como una dicotomía que opone dos modelos de ciudadanía medioambiental, no minusvalora el alcance y los efectos teóricos y prácticos (políticas públicas) de la reformulación verde realizada por algunos defensores de la ciudadanía liberal clásica. Tampoco pretende configurar dos mundos de ciudadanía escindidos, sin contacto alguno, de tal suerte que las dimensiones que ubicamos en uno $u$ otro sean radicalmente excluyentes y no existan espacios intermedios o puntos de conexión.

La política verde y su concepto de ciudadanía ecológica, de la que aquí ofrecemos nuestra específica versión en clave igualitarista, no es antiliberal sino antineoliberal (Murria Rothbard, Robert Nozick), una postura que no debe diluirse con etiquetas de tipo "postliberal" ${ }^{34}$. Sin embargo, nuestro objetivo es emplear dos tipos ideales (que, por tanto, nunca se dan en estado puro en la práctica) para mostrar los muy estrechos límites de la concepción liberal y, junto a ello, exponer la ruptura teórica que promueve una concepción ecosocialista para superarlos.

Podemos sintetizar brevemente las principales discrepancias entre ambas ciudadanías, siguiendo el Cuadro 1.

\footnotetext{
${ }^{34}$ Robyn EcKersley, The Green State, MIT Press, Cambridge, 2004, p. 138.
} 


\begin{tabular}{|c|c|}
\hline $\begin{array}{c}\text { CIUDADANÍA } \\
\text { LIBERAL MEDIOAMBIENTAL }\end{array}$ & $\begin{array}{c}\text { CIUDADANÍA } \\
\text { ECOLÓGICO-IGUALITARIA }\end{array}$ \\
\hline Espacio Político: Estado-Nación & Espacio Político: Huella Ecológica \\
\hline Sustentabilidad Débil & Sustentabilidad Fuerte \\
\hline Antropocentrismo Fuerte & Antropocentrismo Débil \\
\hline Naturaleza: Valor Intrumental & Naturaleza: Valor Intrínseco \\
\hline Neutralidad Estatal & Vida Buena \\
\hline Derechos & Obligaciones \\
\hline Generaciones Presentes & Generaciones Presentes y Futuras \\
\hline Mercado & Comunidad \\
\hline Intereses & Virtudes Cívicas \\
\hline Democracia Representativa & Democracia Republicana \\
\hline Libertad & Igualdad \\
\hline Necesidades Sociales & Necesidades Básicas \\
\hline Recursos & Capacidades \\
\hline Capitalismo Verde & Ecosocialismo \\
\hline Políticas Públicas & Políticas y Reformas Estructurales \\
\hline Esfera Pública & Esfera Pública y Privada \\
\hline Nacionalismo de Estado & Federalismo \\
\hline Monismo Cultural & Pluralismo Cultural \\
\hline Razones & Razones y Emociones/Sensibilidad \\
\hline Educación Técnica & Educación Ciencias/Humanidades \\
\hline
\end{tabular}

Cuadro 1. Tipos de Ciudadanía Medioambiental.

En primer lugar, la ciudadanía ecológica posee una noción de espacio político de generación de obligaciones alternativo al liberal: la huella ecológica. Es preciso resaltar la diferencia cualitativa entre este nuevo espacio y el reducido ámbito del Estado nacional, no solo por la estrechez territorial de este último, sino por la idea subyacente en él de que solo la identidad nacional implica responsabilidad (nacional) y, por lo tanto, la única justicia posible es de base estatal-nacional y esta última abre la única vía transitable hacia la justicia global. La 
asunción básica de esta posición — que articula estatalismo y nacionalismo (de Estado) - tal y como encontramos, por ejemplo, en la más elaborada teorización de esta perspectiva, la de David Miller, es que solo la responsabilidad nacional faculta a la gente a asumir responsabilidades respecto a los sacrificios que la justicia puede requerir, más que postular esos sacrificios como impuestos por una institución (supranacional) cuya autoridad no pueden reconocer ${ }^{35}$.

Por el contrario, la huella ecológica constituye, como vimos, un espacio político global, no territorial, que se erige como fuente de cálculo del exceso de ocupación e impacto medioambiental y de la obligación de reducir la injusticia que supone la distribución desigual de ese impacto (consumo y polución). Esto no quiere decir, en modo alguno, que la nacionalidad y las obligaciones con los connacionales sean de todo punto irrelevantes y la única perspectiva pensable sea la del cosmopolitismo. Pero sí que el espacio de obligación debe ser ampliado y, a la vez, reformulado de modo cualitativo en otra dimensión diferente. Este espacio de impacto ecológico funda normativamente una comunidad global de obligación entre seres humanos iguales, sobre la base de una biosfera finita y vulnerable, que debe ser preservada para las futuras generaciones y cuyos costes de preservación deben ser equitativamente distribuidos en la generación presente.

La ciudadanía ecológica construida a partir de la huella ecológica permite avanzar desde la mera obligación moral hacia una obligación de estricta justicia, como exigencia de vivir equitativamente dentro de los límites de la naturaleza. Justicia que requiere, en el caso de la generación actual, ir más allá de los principios genéricos de los procedimientos democráticos de discusión y consenso en dos sentidos: 1) estableciendo y concretando la métrica de la redistribución (criterios de medida de la igualdad), habida cuenta del pluralismo ideológico y cultural como rasgo estructural permanente; y 2) seleccionando el nivel de gobierno apropiado (diverso nivel de participación, exigibilidad y control en la toma decisiones) considerando el pluralismo de formas de gobierno de los diferentes países $^{36}$. Justicia que implica además, en el caso de la equidad entre generaciones presentes y futuras, pensar en términos no solo de espacio global común sino de auténtico patrimonio común que debe ser preservado mediante diversas medidas de prevención (representación de las futuras generaciones,

${ }^{35}$ David Miller, National Responsibility and Global Justice, Oxford University Press, Oxford, 2007, p. 269.

${ }^{36}$ David Crocker, Ethics of Global Development, Cambridge University Press, Cambridge, 2008, passim. 
monitoreo del patrimonio natural y cultural, incentivos de cooperación y responsabilidad, etc.) $)^{37}$.

Este espacio político-ambiental exige a su vez, como hemos visto, ir más allá de un concepto de sustentabilidad en sentido débil (aquel que solo considera la pérdida de capital total, postulando que el capital natural puede sustituirse por el capital humano y tecnológico), para defender un concepto fuerte de sustentabilidad. Según este concepto la pérdida de capital natural es irreversible. Por las múltiples funciones vitales y ecológicas (y no meramente económicas) que este desempeña para los seres humanos es insustituible. Esta perspectiva en sentido fuerte de la sustentabilidad implica una valoración no esencialista, pero intrínseca, y un compromiso ético-político con la naturaleza no humana que debilita el primer término y refuerza el segundo del sintagma "capital natural". Esto posee consecuencias de relieve no desdeñable porque excluye, como ya hemos apuntado, tanto el antropocentrismo fuerte, que establece una relación de sujeto-objeto con la naturaleza (biosfera) y pone las bases del paradigma de la explotación/dominación ilimitada; como el biocentrismo que ignora que el ser humano es el único agente moral del planeta y está sujeto a una responsabilidad moral en cuanto interviene masivamente sobre la naturaleza ${ }^{38}$.

Ahora bien, la ciudadanía ecológica o ecosocialista aun cuando es postulada desde un antropocentrismo débil, contiene una idea de vida buena explícita (si bien no transparente ni cerrada, sino plural, compleja, contestable y con varias versiones posibles en disputa) y con ello se distancia de la engañosa afirmación liberal de la neutralidad estatal ante las diversas ideas de bien. Engañosa, pues esta última esconde a fin de cuentas una idea implícita de vida buena, que podríamos enunciar así: antropocentrismo fuerte, sustentabilidad débil.

Esta faceta de la ciudadanía ecológica puede formularse también como reconstrucción teórica de la idea del yo: frente a un yo liberal, descontextualizado, solipsista y competitivo, la ciudadanía ecológica ofrece una idea relacional del yo doblemente contextualizada, tanto en la comunidad global de los seres humanos presentes y futuros (igualdad y responsabilidad), como en la naturaleza considerada como biosfera (finitud y vulnerabilidad).

En virtud de los dos argumentos anteriores, bien se desprende que la ciudadanía ecológica se formule, sobre todo, en términos de obligaciones - a diferencia de la liberal con su énfasis en nuevos derechos o derechos de cuarta gene-

${ }^{37}$ WeIss, In Fairness of Future Generations, p. 391.

${ }^{38}$ Riechmann, Un mundo vulnerable, passim; Jorge Riechmann, Biomímesis, Catarata, Madrid, 2006, passim. 
ración prosiguiendo la ya clásica escala de Marshall (derechos personales, políticos, sociales) - Como se ha apuntado, "ya no constituye un sinsentido preguntar si el estado de la naturaleza extrahumana... puede plantearnos algo así como una exigencia moral, no solo en razón de nosotros, sino también en razón de ella y por derecho propio"39. Lo que habilita, por cierto, la posibilidad de formular una suerte de "derechos de la naturaleza" (Constitución de Ecuador), siempre que esto no se realice mediante su constitución en sujeto jurídico unitario, holístico, o de modo dogmático, no mediado por el pluralismo y la deliberación.

La indeterminación y contingencia de la idea de sustentabilidad (que debe ser debatida y traducida en políticas públicas mediante decisiones políticas) se extiende a la ciudadanía ecológica que de ella se deriva, y conduce a su radical politización (y su irreductibilidad a la economía, la ética o al derecho), esto es, a su conversión en objeto de discusión, decisión y contestación. Y, a su vez, esta politización excluye por definición toda tentativa eco-autoritaria o eco-tecnocrática de excepcionalismo, como las que han menudeado en la historia de la teoría ecologista, desde William Ophuls ${ }^{40}$ hasta David Shearman y Joseph WayneSmith ${ }^{41}$ pasando por el reciente "we need a more authoritative World" de James Lovelock en The Guardian.

$\mathrm{El}$ argumento se reitera, si bien con menor fuerza cada vez: la extrema gravedad de la crisis ecológica volvería inadecuada su gestión democrática (por lenta, ineficaz, deudora de las preferencias prejuiciadas y desinformadas del electorado, etc.), exigiendo una suerte de estado de guerra o de estado de excepción con la limitación drástica de la libertad individual y una mayor centralización en la toma de decisiones.

Pues bien, de lo hasta aquí argüido se deduce todo lo contrario: dada la naturaleza política, contestable e indeterminada de ciudadanía ecológica, resulta imposible la ubicación de un contenido ético-político, sustantivo, dogmático e indisputable como fundamento de las instituciones y las políticas públicas. Como antes se ha subrayado, la sustentabilidad es un concepto político normativo - no (solo) científico - y por lo tanto debe ser fijado, concretado y decidido (y contestado) democráticamente por los ciudadanos. Estos tiene que optar colectivamente, decidir y revisar qué costes y qué riesgos están dispuestos a asumir y cuáles no, cuántos recursos van a dedicar y a qué fines, qué modelo de desarrollo

${ }^{39}$ Jonas, El Principio de Responsabilidad, p. 35.

${ }^{40}$ William Ophuls, Ecology and the Politics of Scarcity, Freeman, San Francisco, 1977, passim.

${ }^{41}$ David Shearman, Joseph WaYne-Smith, The Climate Change Challenge and the Failure of Democracy, Greenwood Press, Westport, 2007, passim. 
dentro de los límites de la biosfera elegirán entre los varios posibles, etc. Repitámoslo: la relación entre sustentabilidad y política, entre sustentabilidad y democracia, no es un nexo contingente sino intrínseco, teórico y conceptual. Habida cuenta de la complejidad de su propio concepto; estructurado en torno a los tres ejes mencionados: sociedad global desigual, biosfera vulnerable y generaciones futuras amenazadas. De esta manera, una lectura puramente técnicocientífica de la sustentabilidad resulta, por definición, inadmisible.

Esto reenvía directamente, sin embargo, a la necesidad de cambios en el sistema político democrático tal y como se encuentra institucionalizado en la actualidad; esto es, demanda no solamente nuevas políticas públicas (medioambientalismo), sino también reformas políticas institucionales de gran calado (ecologismo). Ante todo, requiere superar la interpretación minimalista (schumpeteriana) de la democracia representativa como dispositivo único de agregación de las preferencias dadas (o expresadas), de elección y control retrospectivo por parte de los ciudadanos.

La democracia debe ser ampliada y profundizada en varias direcciones. En primer lugar, extrayendo todas las muy exigentes implicaciones normativas de la representación misma, evaporadas en la lectura minimalista (información no distorsionada, igualdad mínima de recursos y control de los grupos de interés que evite asimetrías de poder que se traduzcan en asimetrías políticas, elecciones realmente libres y competitivas, etc. $)^{42}$. Quizás el elemento más novedoso a estos efectos sea la problemática (ética y epistemológica) representación de los "Otros" excluidos en la política representativa ordinaria (la naturaleza y las generaciones futuras), para pasar, también aquí, en el sentido de Anne Phillips, de una "política de las ideas" a una "política de la presencia"43.

En el caso de la naturaleza, consideramos que la sustentabilidad no apela a un espacio natural prístino que hable con transparencia y mucho menos con voz única (aunque esto es, por cierto, una característica que comparte con los seres humanos, pues no se olvide que tampoco estos constituyen "lugares de certeza epistemológica") ${ }^{44}$. Por ello solo podemos dar voz a la naturaleza: 1) sabiendo que los humanos la hemos construido parcialmente con nuestra intervención material y discursiva; 2) que su representación se hará por fuerza a través de

${ }^{42}$ Ramón MÁIz, "Deliberación e inclusión en la democracia republicana": REIS, n. ${ }^{\circ} 113$ (2006), pp. 10-48.

${ }^{43}$ Anne PhILLIPS, The Politics of Presence, Clarendon, Oxford, 1995, passim.

${ }^{44}$ Latour, Politics of Nature, passim. 
actores como proxies o trustees (partidos, movimientos, nuevas circunscripciones medioambientales, ONG, expertos, etc.) que, sin privilegio alguno de ser la voz verdadera de la naturaleza, aporten al debate público y confronten con otros actores el punto de vista de esas dimensiones excluidas; 3) y de la mano, además, de una democracia que incorpore procesos deliberativos de decisión, pues "la deliberación inclusiva requiere formas de representación más inclusivas y nuevos procesos y reglas de decisión ecológicamente sensibles" ${ }^{45}$.

Un buen ejemplo de como todo esto puede concretarse institucionalmente podemos verlo en la aplicación del principio de precaución. Este principio suele ser criticado desde posiciones liberales medioambientalistas o incluso republicanas por poseer un sesgo intrínsecamente fundamentalista - no interferir con la naturaleza - que se traduce en la proliferación de no-decisiones (económicas, técnicas, de investigación científica) ante la mera presencia de riesgo. Esto impide la necesaria gestión del riesgo, omnipresente en toda decisión, y por lo tanto deviene paralizante para la acción ${ }^{46}$. Ahora bien, desde la perspectiva que aquí se plantea cabe una lectura bien diferente: el principio de precaución se puede adoptar como una regla de decisión alternativa al análisis coste-beneficio dominante. El problema fundamental de este último es que atiende exclusivamente a la eficacia económica, de modo que solo toma en consideración las preferencias dadas de los ciudadanos. Una perspectiva que resulta extraordinariamente problemática en cuestiones medioambientales, necesitadas de información no distorsionada y debate irrestricto, exento de dominación.

Por el contrario, una interpretación política del principio de precaución proporciona una valiosa orientación normativa en situaciones de incertidumbre y no simple riesgo, sugiriendo pautas institucionales y procedimentales. Posibilitaría, así, un proceso de decisión precavida (precautionary decision making, precautionary appraisal process $)^{47}$, que incorpora diversos requisitos para un adecuado mapa decisional: independencia de los grupos de interés, examen de los grados de incer-

45 "Inclusive deliberation also demands more inclusive forms of representation and new, ecological sensitive procedures and decision rules". [Una deliberación inclusiva requiere también de formas de representación más inclusivas y nuevos procesos y normas de decisión con sensibilidad ecológica]. ECKersley, The Green State, p. 137.

${ }^{46}$ Véanse Cass Sunstein, Laws of Fear. Beyond the Precautionary Principle, Cambridge University Press, Cambridge, 2005, passim; SEN, The Idea of Justice, passim; GIDDENs, The Politics of Climate Change, passim.

${ }^{47}$ Carolyn Raffensperger, Joel Tickner (eds.), Protecting Public Health and the Environment, Island Press, Washington, D.C., 1999, passim; Andrew STIRLING, "Participation, Precaution and Reflexive Governance for Sustainable Development", en Adger, JoRdan (eds.), Governing Sustainability, pp. 193-225. 
tidumbre, consideración de efectos indirectos, evaluación de efectos en ciclos completos, incorporación de conocimiento relevante, discusión explícita sobre los límites apropiados a la experimentación, prueba, evidencia y análisis, etc. ${ }^{48}$.

Esto nos conduce, en segundo lugar, a la necesidad ineludible de completar con exigencias de democracia republicana los mecanismos representativos con otros de participación (a escala local, referéndum, iniciativa popular, repertorio disruptivo de movilización y protesta, etc.); de deliberación (consejos ciudadanos, paneles cívicos, minipopulus, encuestas deliberativas, etc.); y de inclusión (de sectores vulnerables o marginados y más afectados - y aún de los pasivos "stakeholders"- por los efectos de la baja calidad medioambiental, polución, cambio climático, etc.).

En razón de la necesidad de cambios estructurales y de políticas, así como de hábitos y valores que la idea de sustentabilidad conlleva, bien se entiende que la ciudadanía ecológica muestra una especial sintonía con la idea de deliberación política $^{49}$. Recordemos que esta se caracteriza precisamente no por agregar preferencias exógenas, sino por: a) mejorar (de modo endógeno) las preferencias previas de los ciudadanos, mediante discusión publica exenta de coacción y con información no distorsionada, y b) poseer carácter decisorio, vinculante y no meramente consultivo. En concreto, la democracia deliberativa aporta, entre muchos otros, tres elementos fundamentales para afrontar los riesgos medioambientales: diálogo irrestricto, inclusividad y aprendizaje social ${ }^{50}$.

Cuando hablamos de "radical indeterminación" (Claude Lefort) aplicado a la sustentabilidad, debemos entender ésta en toda su extensión: 1) se trata de un proceso abierto, revisable y contingente, cuyo alcance y contenido en cada contexto no puede cerrarse; y 2) la apertura democrática del concepto elimina por definición las "referencias de certidumbre", y nada garantiza los resultados: que los ciudadanos adopten una política ecológica u otra, entre las muchas posibles, o incluso que no acepten ninguna. Se trata de una lucha política incierta por la hegemonía en la que se solventa el eventual triunfo (siempre parcial y en precario) del ideal cívico ecológico. Esto reclama, más allá de la esfera comunicativodiscursiva, la necesidad de una dinámica permanente de conexión y retroalimentación entre el ideal teórico-normativo, por una parte, y el activismo de la lucha ideológica y el repertorio de movilización política ecologista, por otra.

${ }^{48}$ Stirling, "Participation, Precaution and Reflexive Governance for Sustainable Development”, p. 205.

${ }^{49}$ Manuel ArIAS, Sueño y mentira del ecologismo, Siglo XXI, Madrid, 2008, passim.

${ }^{50}$ Robyn ECKersLey, Environmentalism and Political Theory, SUNY, New York, 1992, passim. 
Estos rasgos iniciales de la ciudadanía ecosocialista se prolongan, además, en otros componentes de capital relieve. En efecto, un factor constitutivo básico de la ciudadanía ecológica viene dado por el principio de comunidad. Ahora bien, como ya debe ser evidente a estas alturas, en razón del concepto constructivista realista de naturaleza que le es propio ${ }^{51}$, debe disiparse de entrada cualquier equívoco respecto a que esta noción de comunidad suponga un retorno al Paraíso Perdido de nostalgia horaciana, al mito de la Reconciliación del ser humano con la naturaleza. Sin embargo, tampoco deben desconocerse las decisivas razones sociales y políticas que explican la recurrente imagen rousseauniana del retournons, de la vuelta a la Naturaleza, en el ecologismo. Tal y como Theodor Adorno (19031969) escribió con extraordinaria lucidez en su día:

Mientras un progreso utilitarista y romo siga violentando la superficie de la Tierra, no podremos librarnos del todo de la idea de que cuanto hay más acá o con anterioridad del rumbo actual, es mejor y más humano precisamente por haberse rezagado...La imagen de la naturaleza sobrevive porque al ser negada completamente en los artefactos se hace ciega para aquello que estaría más allá de la sociedad capitalista, de su trabajo y sus mercancías. La belleza natural es la alegoría de este más allás ${ }^{52}$.

La idea comunitaria propia de la ciudadanía ecológica aquí postulada debe ser entendida no solo como una comunidad ampliada de seres humanos y naturaleza, con fronteras difusas y cambiantes; sino elaborada además a partir de dos asunciones: 1) que la naturaleza es a la vez parcialmente producida por los seres humanos material y simbólicamente; y 2) que no existe fusión o reconciliación posible, inmersión holista en la naturaleza sino tensión, conflicto, riesgo y trabajo. Por ende, se trata de una comunidad político-discursiva y moral de obligación igualitaria intergeneracional e intrageneracional. Esto es, una comunidad que resulta cualitativamente distinta de la propia del comunitarismo liberal. A diferencia de este, está pensada desde el principio del mutualismo igualitario, de la reciprocidad no instrumental, en su sentido fuerte (strong reciprocity) ${ }^{53}$, como alternativa a la maximización del beneficio material en el corto plazo. Se trata

${ }^{51}$ Ted Benton, Ian Craib, Philosophy of Social Science, Palgrave, New York, 2001, passim.

${ }^{52}$ Theodor Adorno, Teoría estética, Taurus, Madrid, 1971, p. 96.

${ }^{53}$ Herbert GinTis, Samuel Bowles, Robert T. Boyd, Ernst FeHR (eds.), Moral sentiments and material interests. The foundations of Cooperation in Economic Life, Harvard University Press, Cambridge, 2005, passim. 
aquí del principio, negador del mercado y su reciprocidad utilitaria, según el cual se asume una obligación no por lo que se pueda obtener cumpliéndola, sino por la necesidad que el otro tiene de la misma ${ }^{54}$.

De ahí que el énfasis en la ciudadanía ecológica se sitúe en la igualdad más que en la libertad, esto es, en el principio de que los bienes y las males, los beneficios y las cargas en la vida de las personas deben ser lo más equiparables posible. Los menos dotados de recursos son más vulnerables al cambio climático, pero, no se olvide, no son pobres debido al cambio climático, sino a estructuras sociales y económicas injustas. Frente a un falso universalismo - el todos somos responsables - , una perspectiva igualitaria atribuye asimétricas responsabilidades, poniendo su acento redistributivo en quienes más huella ecológica dejan sobre el planeta, más recursos consumen, más combustibles fósiles emplean, quienes mayores índices de emisiones de carbono generan, etc.

Además, hay otros argumentos decisivos a estos efectos igualitarios: 1) la complejidad de los conocimientos requeridos por la inteligencia socio-ecológica desborda las capacidades individuales y precisa de un trabajo colectivo y en red $^{55}$, el cual solo es pensable desde la empatía y la igualdad; y 2) la ya apuntada necesidad de trascender el ámbito del Estado-nación y avanzar hacia formas federales de autogobierno y cooperación, sobre lo que volveremos más adelante, sitúa la igualdad entre clases sociales y entre regiones y países como un objetivo normativo irrenunciable. La razón es clara: la desigualdad genera, entre otras cosas, una intolerable asimetría estructural en la capacidad de negociación entre las partes y, por tanto, constituye un obstáculo sistémico para la justicia en la distribución de los males y los bienes, los sacrificios y las recompensas, los costes y los beneficios entre clases y naciones.

Pero aún más, la ciudadanía ecológica resulta inseparable de una crítica sustantiva al capitalismo en cuanto éste se funda en el sometimiento de la naturaleza a los imperativos de valorización del capital, los cuales empujan hacia una expansión económica indefinida en una biosfera finita ${ }^{56}$. Razón por la cual la ciu-

${ }^{54}$ Gerald Allan CoHen, Why not socialism?, Princeton University Press, Princeton, 2009, passim Félix Ovejero, Roberto Gargarella (eds.), Razones para el socialismo, Paidós, Barcelona, 2001, passim.

${ }^{55}$ Daniel Goleman, Ecological Intelligence, Penguin, London, 2009, passim.

${ }^{56}$ Martin O' Connor, Is Capitalism Sustainable?, Guilford Press, New York, 1994, passim. James O' Connor, Causas Naturales. Ensayos de marxismo ecológico, Siglo XXI, Madrid, 2001, passim. Ted Benton, "Marxism and Natural limits": New Left Review, vol. 178 (1989); Nature, Social Relations and Human Needs. Essays in honour of Ted Benton, Palgrave, London, 2009, passim. Jorge Riechmann, La habitación de Pascal, Catarata, Madrid, 2009; y también de este autor las ya citadas Un mundo vulnerable (2004) y Biomémesis (2006). 
dadanía ecológica y la noción igualitarista de sustentabilidad muestran una incompatibilidad de raíz con la lógica del capital y poseen, por el contrario, una indisoluble vinculación teórica y conceptual con la idea igualitaria socialista.

De hecho, desde la perspectiva que aquí venimos sosteniendo, el capitalismo resulta medioambientalmente destructivo. La presión competitiva por maximizar los beneficios aboca a las empresas a ignorar los costes medioambientales derivados de su actividad (residuos, polución, destrucción de recursos naturales). Por decirlo en los desacomplejados términos de Milton Friedman (1912-2006) en Capitalismo y Libertad: "La responsabilidad social de la empresa consiste en aumentar sus beneficios" ${ }^{57}$. Pero, además, porque los recursos no renovables resultan sistemáticamente consumidos a muy bajo precio, debido al omnipresente mecanismo del descuento del futuro, es decir, por la desconsideración de su valor para las generaciones futuras (y de ahí la insostenibilidad de su modelo de crecimiento). El capitalismo, además, en razón de su cultivo del estrecho autointerés, de su horizonte a corto plazo, de la adopción de decisiones fundamentales en ámbitos privados ajenos a cualquier control público, etc., se muestra especialmente inadecuado para la justicia intergeneracional que supone la sustentabilidad ${ }^{58}$.

La ciudadanía ecologista reformulada desde la igualdad se diferencia de la ciudadanía liberal medioambiental en que postula la necesidad de cambios estructurales profundos en el sistema económico y modificaciones asimismo de gran calado en la cultura política dominante (y de la cultura tout court) que comparten los ciudadanos.

En lo que al primer aspecto se refiere, resulta preciso destacar que la ciudadanía ecológica se distancia abiertamente de los postulados del "mediambientalismo de mercado" (market environmentalism) o el capitalismo verde (green capitalism). En su perspectiva, desde luego, no se trata solo ni fundamentalmente de incentivar que los actores privados persigan sus intereses particulares en modos que simultáneamente promuevan la sustentabilidad (débil) ${ }^{59}$. Sino, además y sobre todo, de planificar y regular la economía de mercado en aras de mantenerla dentro de ciertos límites, además de gestionar la demanda de los consumidores en lugar de excitarla de modo compulsivo, y de ahí la necesidad perentoria de una gobernanza de la sustentabilidad ${ }^{60}$.

${ }^{57}$ Milton Friedman, Capitalismo y libertad, Rialp, Madrid, 1966, p. 132.

${ }^{58}$ Eric Olin Wright, Envisioning Real Utopias, Verso, London, 2010, p. 54.

${ }^{59}$ Steven BernsteIn, The Compromise of Liberal Environmentalism, Columbia University Press, 2001, passim.

${ }^{60}$ Adger, JoRdAn, Governing Sustainability, passim. 
Esta perspectiva se opone, ante todo, a la vulgata del neoliberalismo, o sea, desregular los mercados, privatizar el sector público, desregular los flujos financieros, reducir los impuestos a las empresas, recortar los derechos sociales y sindicales y erosionar el Estado de bienestar. Pero además, una estrategia de ciudadanía ecológica se inserta en un marco de reformas públicas incompatible con mecanismos como la compraventa de derechos de emisión en el mercado del carbono, que subyace tras estrategias en boga como cap and trade o personal carbon allowances.

En efecto, este tipo de mecanismos suponen una mercantilización del medio ambiente que refuerza la lógica de la desregulación. Mediante la creación de nuevos mercados paralelos, la mercantilización llega a alcanzar temas de alto riesgo como el efecto invernadero y el calentamiento global. Asimismo estos procedimientos expulsan de la agenda política las cuestiones centrales de la justicia y la igualdad, tanto en el consumo de recursos naturales como en la producción y control de residuos (qué peso se otorga a las futuras generaciones y qué remedios damos a las enormes desigualdades económicas en la presente generación). Otra consecuencia es que se convierte al sector privado (empresas, fondos de inversión) en el principal agente de gobierno y control del cambio climático: los Estados, la Unión Europea, los acuerdos o foros internacionales, por no hablar de los ciudadanos, quedan rebajados a meros consumidores desinformados ${ }^{61}$.

Pero en segundo lugar, como quiera que el capitalismo fomenta de modo sistemático una cultura de consumo compulsivo, acompañada de una "asimetría de información" (Joseph Stiglitz), la ciudadanía ecológica debe incorporar —al hilo de sus principios de responsabilidad e igualdad - un ethos específico de valores, hábitos, actitudes y sensibilidades. Por una parte, las políticas públicas de gestión de la demanda (editing choice) deben abandonar el despropósito ecológico de pseudo-dogmas como la "soberanía del consumidor" e implementar incentivos positivos y negativos de orientación del consumo, tanto a las empresas como a los ciudadanos (por ejemplo, en lo que concierne a emisiones de $\mathrm{CO}_{2}$, energías alternativas, reciclado, reutilización, contaminación, etc.). Por otra parte, estas medidas deben ser acompañadas de cambios en los estilos de vida y en las pautas de comportamiento individuales. También aquí, como mostró hace tiempo el feminismo desbordando sus iniciales objetivos, lo personal es político.

Las necesidades y los límites, reformulados desde nuestra perspectiva de la sustentabilidad, no afectan solo a las decisiones colectivas (políticas públicas,

${ }^{61}$ Mike Hulme, Why we disagree about climate change, Cambridge University Press, Cambridge, 2009, p. 303. 
reformas institucionales) sino al propio ámbito privado de la ciudadanía. Ya hemos visto cómo el cambio estructural por si solo no resulta suficiente para alcanzar la igualdad; también se requiere un cambio en el ethos social, en las actitudes y sentimientos que los seres humanos tienen unos con otros en la vida cotidiana (empatía), para producir social y políticamente la igualdad mediante la ya aludida reciprocidad no instrumental (strong reciprocity) ${ }^{62}$.

Pues bien, lo mismo sucede con la sustentabilidad: la idea de límite debe ampliarse para incluir una perspectiva de autolimitación individual, de nuevas pautas y estilos de vida desde la autolimitación y la suficiencia ${ }^{63}$, una nueva sensibilidad ante la naturaleza, un ethos ecológico que resulta indispensable para construir políticamente, a partir de los ciudadanos en sus decisiones privadas, en las relaciones entre ellos y con la naturaleza, una sociedad sustentable. Del mismo modo que no puede sobrevivir una democracia sin demócratas, no puede alcanzarse una sociedad sustentable basada en una ciudadanía huérfana de inteligencia ecológica ${ }^{64}$, sin los sentimientos morales, sin sensibilidad necesaria para evaluar los impactos de nuestras acciones sobre la biosfera, la salud y los sistemas sociales y, por el contrario, con necesidades expansivas y no comprometida con el reciclado, con un menor (y alternativo) consumo energético, con una movilización electoral y de protesta ante el problema ecológico, etc.

Resulta necesario ser muy claro a este respecto: solamente es posible no plantear la sustentabilidad exclusivamente desde arriba (top down) y en clave negativa o coercitiva, esto es, solo mediante medidas restrictivas y prohibiciones $\mathrm{o}$, aún peor, promoviendo una fundamentalista frugalidad represiva de la siguiente manera: mediante la defensa (desde el debate abierto, la experimentación y los incentivos por parte del Estado mediante la gestión de la demanda), de un nuevo ethos ecológico y sustentable, de nuevos estilos plurales de vida buena, que tengan en cuenta la maleabilidad de las capacidades humanas para el placer y la construcción de necesidades.

Del mismo modo que la utilización exclusiva o dominante de mecanismos de mercado (cap and trade) refuerza la mercantilización del medio ambiente, desapodera a los gobiernos y a los ciudadanos, así como obstaculiza los cambios estructurales necesarios, las políticas públicas medioambientales superficiales o las modas de dar un mero retoque verde a los programas electorales (greening

\footnotetext{
62 Véase nota 53.

${ }^{63}$ Véase nota 24.

${ }^{64}$ Véase nota 55.
} 
policies), refuerza la ilusión de que un perfil bajo de compromiso personal y político resulta suficiente. Esto es, que no son necesarios cambios en los estilos de vida (por ejemplo, de alto consumo de agua, energía y medio de transporte individual) y en las políticas públicas (por ejemplo, políticas de transportes e infraestructuras basadas en la ecuación: cemento + combustibles fósiles $=$ transporte individual de bajo coste). De ahí que, más allá de los necesarios pero insuficientes incentivos institucionales positivos y negativos, la batalla ideológica y el cambio cultural de valores y actitudes resulta absolutamente imprescindible. Pero, por esa misma razón, la ciudadanía ecológica en la práctica resulta inviable sin el ecologismo político como organización e ideología.

De ahí, en fin, por una parte, la necesidad de una educación cívica que promueva una inteligencia ecológica, los conocimientos, valores y actitudes implicados en la sustentabilidad. Ello no requiere, sin embargo, una suerte de adoctrinamiento verde masivo - por más que toda educación comporte, por definición, una posición ético-política y una perspectiva neutral resulte engañosa- cuanto un decidido sesgo ecológico y no solo medioambiental en el qué y el cómo se enseña. En este sentido, por ejemplo, frente a la tendencia dominante en la actualidad a orientar los planes de estudio y las habilidades pedagógicas hacia materias técnicas y una creciente obsesión con las "necesidades del mercado", la ciudadanía ecológica requiere una educación que concilie el conocimiento científico con las capacidades que proporciona el estudio de las artes y las humanidades. Respecto a la centralidad pedagógica de estas últimas la razón es evidente: la ciencia no puede crear fines, solo proporciona medios con los que alcanzarlos, y menos aún enseñarlos a los seres humanos ${ }^{65}$. Y las humanidades ${ }^{66}$ proveen de al menos tres habilidades esenciales para la ciudadanía ecológica: 1) la capacidad de cuestionarse desde la reflexión crítica y la relevancia social de los problemas; 2) la capacidad de desarrollar empatía con los demás seres humanos (y no humanos sintientes) y con el medio ambiente; y 3 ) la capacidad de trascender los lazos locales y nacionales para atender los problemas de la sociedad global.

Pero, por otra parte, el ethos propio de la ciudadanía ecológica requiere algo más: modular una perspectiva centrada exclusivamente en los argumentos y las razones, con el cultivo de emociones y sensibilidades apropiadas (empatía con

${ }^{65}$ Albert Einstern, “Why Socialism?”: Monthly Review (May 1949).

${ }^{66}$ Martha C. Nussbaum, Not for Profit: Why Democracy Needs the Humanities, Princeton University Press, Princeton, 2010, p. 32. 
todos los seres sintientes, fraternidad igualitaria con las generaciones presentes y futuras, indignación ante la destrucción del paisaje, etc.). No vamos a insistir ahora en la relevancia política de las pasiones, la cual ya hemos analizado con cierto detalle en otro lugar ${ }^{67}$, pero sí debemos apuntar, al menos, que la ciudadanía ecológica urge el abandono de una perspectiva muy extendida que insiste en una natural predisposición femenina para las emociones ante la naturaleza (véase cuadro 1). Por el contrario, la sustentabilidad requiere un tránsito desde la imaginería de la Madre Naturaleza y sus derivados (mothering Earth), esto es, desde una noción hiperfeminizada de cuidado, a la adopción de una perspectiva cívica ecológica que compromete políticamente (con razones y emociones apropiadas) tanto a hombres como a mujeres en una común tarea ${ }^{68}$.

Finalmente, en razón del espacio político que propugna, la perspectiva cívico-liberal ambientalista es profundamente estatalista y, en consecuencia, promueve como única base para generar obligaciones de justicia medioambiental, de asumir el costo y los sacrificios que implican las políticas ambientales, los muy cerrados lazos de la comunidad nacional ${ }^{69}$. Esto implica, por una parte, la adopción hacia el exterior de una perspectiva global de "relaciones internacionales", y por otra, hacia el interior de cada país, un estrechamiento comunitarista y monocultural de la nación. El fracaso parcial de los acuerdos e instancias supranacionales (Kyoto, Copenhague) ha reforzado estas perspectivas, promoviendo nuevas narrativas ecológicas nacionalistas (nation-building naratives) ${ }^{70}$.

Sin embargo, la ciudadanía ecosocialista apunta en dirección muy diferente: hacia una perspectiva multicultural/multinacional, por una parte, y federal, por otra. El reconocimiento de la pluralidad de las valoraciones sobre los bienes (recursos, capacidades) y los males (residuos) implicados en el debate de la sustentabilidad y la concreción de los principios de redistribución inter e intrageneracional, se traduce en el reconocimiento de que unos y otros son contextuales y no existe un principio de justicia universal incontestable, válido para todo tiempo y lugar. Pero en esta perspectiva, ante el riesgo global de la crisis ecológica, y en virtud del nuevo espacio político que se propugna en esta ciudadanía, la alternativa se muestra como una superación del marco del Estado nacional y las

\footnotetext{
${ }^{67}$ Ramón MÁzz, "The Political Mind and its Other”, en Marcos EngeLKEn-JORgE et al. (eds), Politics and Emotions, VS Verlag für Sozialwissenschaften, Wiesbaden, 2011, pp. 29-70.

${ }^{68}$ Sherilyn MACGregor, Beyond Mothering Earth, UBC, Toronto, 2006, passim.

${ }^{69}$ MiLler, National Responsibility and Global Justice, passim.

${ }^{70}$ David Hetherington, What's the Story? Nation-building Narratives and Climate Politics, Policy Network, London, 2010, passim.
} 
relaciones internacionales, en una perspectiva ajena tanto a una difusa comunidad moral internacional, cuanto a un centralizado gobierno mundial.

La superación de esta última perspectiva resulta especialmente necesaria por varias razones: 1) la consideración de que el pluralismo cultural es un hecho y además un valor que promueve una pluralidad de interpretaciones de bienes y obligaciones hace que la sola idea de una autoridad global dotada de criterio único para todo el planeta sea un sinsentido; y 2) la superación del marco del Estado nacional debe realizarse sin menoscabo de los lazos identitarios (muchas veces, a su vez, internamente plurales) y las garantías políticas que aquél proporciona: control democrático de las decisiones, redistribución de recursos mediante sistemas fiscales progresivos, provisión de derechos sociales y bienestar, etc.

Por el contrario, el reconocimiento de la multiplicidad de identidades y culturas y del entramado de contextos de decisión multinivel en el seno de los cuales debe afrontarse la crisis ecológica, pone en primer plano la potencialidad del ideal federalista ${ }^{71}$ el cual articula autogobierno y gobierno compartido, unidad y diferencia, igualdad y responsabilidad ${ }^{72}$. De hecho, la teoría de la evolución y la biología nos proporcionan modelos heurísticos de gran utilidad para incorporar con toda sustantividad la hipótesis federal. Así, una perspectiva de biomímesis ("Nature knows better": recuérdense los foedera naturae de Lucrecio en De Rerum Natura), reemplazando a la obsoleta imaginería mecanicista ilustrada clásica de "mecanismos", "frenos" y "contrapesos", nos sugiere nuevas imágenes de desarrollo institucional federativos inspirados en la naturaleza, como la simbiosis, la endosimbiosis, la colonia, la diversificación y cooperación entre diversos organismos, etc. ${ }^{73}$. No por azar la teoría política ecologista — que, no se olvide, siempre ha tenido una sensibilidad descentralizadora y multinivel-, y los estudiosos de las políticas públicas medioambientales, en sus desarrollos más recientes, han desechado una perspectiva de "command and control" por otra de gobernanza multicéntrica y en red.

Por mor de estas y otras razones, el ecologismo ha abandonado algunas iniciales tentaciones centralistas y autoritarias de postular una autoridad o gobierno mundial para gestionar el riesgo ecológico. Unos y otros han pasado a defender nuevas formas de descentralización y federalismo, por considerarlas más adapta-

${ }^{71}$ Francisco FernándeZ BueY y Jorge Riechmann, Ni Tribunos: ideas y materiales para un programa ecosocialista, Siglo XXI, Madrid, 1996, p. 191.

${ }^{72}$ Ramón MÁzz, La frontera interior, Tres Culturas, Murcia/Madrid, 2008, passim.

${ }^{73}$ Janine M. BenYus, Biomimicry. Innovation inspired by Nature, HarperCollins, New York, 1997, passim. 
das a contextos política y culturalmente complejos, así como para abarcar diversas configuraciones de ámbitos de decisión, siempre partiendo del gobierno local, la integración de políticas públicas, el pensamiento a largo plazo y el pluralismo cultural ${ }^{74}$. En la política contra el cambio climático, por ejemplo, se ha insistido frente a ineficaces modelos top-down para abordar el calentamiento global (Kyoto), en la necesidad de adoptar una perspectiva multiescalar para adaptarse a los diferentes y específicos riesgos que deben afrontar las distintas comunidades, y de ahí se ha pasado a defender un "global federalism of climate policy". Se vislumbra así un nexo muy prometedor, si bien aún inexplorado, entre la teoría del federalismo y los diseños institucionales emergentes de la gestión descentralizada y multinivel de la sustentabilidad igualitaria.

\section{REFLEXIONES FINALES}

Para terminar cabe añadir que el principio de la sustentabilidad igualitarista y el concepto de ciudadanía ecológica a favor del que aquí argumentamos debe implicar, a nuestro juicio, algunas reformulaciones normativas adicionales de la teoría de las capacidades (Nussbaum, Sen) de no escaso relieve; a saber: el criterio de la satisfacción de las necesidades básicas deberá atender: 1) a la igualdad de oportunidades, en cuanto libertad real para todos los seres humanos de la presente generación, 2) al desarrollo económico desde el respeto de los límites de la biosfera y 3) a la responsabilidad hacia la igualdad de oportunidades y acceso a las ventajas de las futuras generaciones.

Ahora bien, esto requiere repensar radicalmente el listado de las capacidades funcionales humanas básicas, que Nussbaum construye como segundo nivel de su gruesa y vaga concepción de la idea de bien. Del mismo modo que los principios de libertad e igualdad poseen un valor tan decisivo para la vida humana que deben ser extraídos y cualitativamente priorizados como "principios de justicia", por parte de John Rawls (1921-2002), sobre la lista de "bienes primarios", el principio de sustentabilidad no puede verse diluido y rebajado, como anecdó-

${ }^{74}$ W. Neil Adger, Irene Lorenzoni y Kate O’Brien (eds.), Adapting to Climate Change, Cambridge University Press, Cambridge, 2009, passim. David BENSON y Andrew JordAn, "Understanding task allocation in the EU: exploring the value of federal theory": Journal of European Public Policy, vol. 15, n. ${ }^{\circ} 1$ (2008), pp 78-97. Timothy O'Riordan, Environmental Science for Environmental management, Prentice Hall, London, 2000, p. 342. 
tico "poder vivir preocupado por animales, plantas y el mundo de la naturaleza", a un mero apartado más del elenco de capacidades funcionales humanas básicas ${ }^{75}$. La relación sustentable de los seres humanos con la naturaleza, la procura de equilibrio entre la tecnosfera y la ecosfera, no constituye una capacidad funcional humana más entre otras, sino la condición misma de su posibilidad, el inexcusable horizonte de interpretación desde la libertad real y la igualdad de oportunidades, para nuestro tiempo, de todas las necesidades humanas básicas.

Como escribió Agnes Heller:

Los hogares en los que uno realmente vive y habita, obligan. En nuestro mundo del presente absoluto incluso el canto de los pájaros y la sombra del castaño obligan, porque ya no podemos presuponer que estén aquí mañana. ¿Dónde estamos en casa? Cada uno de nosotros en el mundo de nuestro destino autoescogido y compartido $^{76}$.

${ }^{75}$ Martha C. Nussbaum, “Aristotelian Social-Democracy”, en Bruce Douglas, Gerald MARA y Henry RichardSON (eds.), Liberalism and The Good, Routledge, New York, 1991; "Capabilities as fundamental entitlements", en Alexander KaUfMan, Capabilities Equality, Routledge, London/New York, 2006.

${ }^{76}$ Agnes Heller, "Where Are We At Home?": Thesis Eleven, n. ${ }^{4} 1$ (1995), p. 54. 